\title{
Mobile multimedia crowd service cooperation control protocol based on opportunistic wavelet
}

\author{
Wei-hang Shi ${ }^{*}$ and Wei-ping $\mathrm{Li}^{2,3}$
}

\begin{abstract}
In order to improve the quality of multimedia communication and experience, we proposed a crowd service cooperation control protocol based on the opportunistic wavelet model. Firstly, in order to provide real-time and reliable guarantee for mobile multimedia services, we analyze the delay, jitter, and distortion of wireless multimedia streams transmission based on wavelet model. The method of video frame resolution analysis and wavelet scale space is combined to establish a wavelet multimedia mobile analysis model. Secondly, the crowd service plan was proposed to correctly decode and compensate the video frame in the mobile multimedia receiver. Finally, the cooperative control of crowd services was proposed to address the distortion competition problems of the video frame sequence. A set of experimental results demonstrate the capability of video frame quality assurance for mobile multimedia crowd service cooperation control protocol. Another group of comparative analysis of the experiment results fully demonstrate the mobile multimedia crowd service cooperative control protocol can provide the higher system throughput and smaller delay compared with the guarantee mechanism of multimedia quality of service based on wavelet analysis.
\end{abstract}

Keywords: Mobile multimedia, Opportunistic wavelet, Crowd service, Cooperation control

\section{Introduction}

Because of the intense competition in the channel, the transmission bandwidth is limited [1], the video distortion rate is higher [2], the external interference factors are unknown, and the [3] node mobile [4] leads to a serious decline in the quality of multimedia communication. Such a multimedia flow cannot meet the user's viewing needs. Multimedia video frame compensation competition [5] and the randomness of the mobile nodes lead to the quality of multimedia transmission that is restricted by the video distortion and interference. The above factors make the service quality of the mobile network real-time multimedia service delivery cannot be effectively guaranteed.

Also in the mobile network, because the multiple distortion of video frames need to share the same opportunistic compensation [6], coordination video

\footnotetext{
* Correspondence: shiweihangzd@163.com

${ }^{1}$ College of Software Technology, Zheng Zhou University, Zhengzhou, Henan 450002, China

Full list of author information is available at the end of the article
}

frame distortion judgment and the secondary compensation is very important, so the design can effectively use mobile network topology characteristics and real-time multimedia mobile service transmission characteristics improve mobile multimedia services intelligent level, attracting the attention of many researchers in academia and industry $[7,8]$.

The video processing algorithm was mapped onto a learning method to improve machine to the parallel reconfigurable computing architecture [9]. The author of article [10] proposed the service-oriented mobile multimedia cooperative storing, and the delivery scheme to improve the quality of multimedia communication and mobile users, the work efficiency of the cloud platform. The security and privacy issues of multimedia services were investigated in article [11] by studying a newly emerging multimedia-oriented mobile social network, which helps users receive multimedia services not only from their online social communities. The authors of article [12] proposed a hybrid cloud architecture, which serves as a substrate for scalable and fast time-to-market 
mobile multimedia services. The link quality and geographical beaconless OR protocol for efficient video dissemination was presented in article [13] for mobile multimedia Internet of Things, which relies on a beaconless OR approach and uses multiple metrics for routing decisions. The improving map that reduces task scheduling and micro-partitioning mechanism was proposed by the authors of article [14] for mobile cloud multimedia services. The authors of article [15] proposed a dual mode self-adaption handoff mechanism for multimedia services in mobile cloud computing environment to improve handoff quality and minimize utilized bandwidth. An adaptive modulation coding technique with cross-layer mapper module is proposed in article [16] for enhancing the quality of service, which is used to realize the main bottleneck parameters for the multimedia applications such as bandwidth, delay, and throughput. The article [17] studied the home nursing guidance based on the application of multimedia messaging services via mobile phones to support outpatients for the pediatric intestinal colostomy complications.

In this paper, we presented a mobile multimedia crowd service cooperation control protocol based on opportunistic wavelet to improve the quality of multimedia communication and experience. The general architecture of the crowd service cooperation control protocol was proposed by the analyzation of the delay, jitter, and distortion of the wireless transmission of multimedia streams based on the wavelet model. The cooperative control of mobile multimedia crowd service was researched and addressed the problem such as the mobile multimedia receiver cannot correctly decode and compensate for the video frame.

The rest of the paper is organized as follows. Section 2 shows the opportunistic wavelet multimedia mobility model. Cooperative control of mobile multimedia crowd service was discussed in Section 3. The results are shown in Section 4. Finally, Section 5 concludes this paper.

\section{Opportunistic wavelet multimedia mobility model}

In order to provide real-time and reliable guarantee for the mobile multimedia services, we analyzed the delay, jitter, and distortion of the wireless transmission of multimedia streams based on the wavelet model. The method of video frame resolution analysis and wavelet scale space are combined to establish a wavelet multimedia mobile analysis model. In the wavelet scale space, the opportunistic sampling method is used to capture the video frame sequence. In the opportunistic wavelet domain, the opportunistic perception method is used to capture the moving sequence of multimedia.

In the process of wavelet transform, we use the opportunistic wavelet transform to analyze the video frame sequence. The analysis result is helpful to process the jitter dispersion of multimedia mobile transmission. Large delay jitter of the video frame of the static multimedia transmission severely reduces the quality of the multimedia broadcast. The video frame sequence of the dynamic multimedia transmission is easy to be disorderly and aggravate the jitter.

In the opportunistic wavelet multimedia mobile model, the opportunistic video frame sequence is reconstructed by the opportunistic wavelet linear transform. This sequence is performed by opportunistic recombination resolution. This can obtain the opportunistic mobile multimedia video frame wavelet scale space. In the scale space and opportunistic wavelet domain, the moving scale coefficients and the opportunistic wavelet weights are adjusted in real time. This can generate a new type of mobile multimedia video frame.

Symbol $F$ denotes the multimedia original data video frame sequence. In order to establish the opportunistic wavelet modeling in multimedia communication, we first convert the $F$ into $G$ sequence, as shown in the formula (1).

$$
G=\sum_{j=m(i-1)+1}^{k i} \delta F_{j}, i=1,2, \cdots\left[\frac{k}{j}\right]
$$

Here, $\delta$ is used to describe the opportunistic weight of the original video sequence. $k$ represents the length of the original video frame sequence. $j$ represents the size of the video frame. $i$ represents a linear video frame number. Symbol $m$ represents linear video frame size.

The opportunistic wavelet model is based on opportunistic wavelet linear transformation, which is used to obtain the opportunistic wavelet function $O_{W}$ and the opportunistic scaling function $S_{W}$, as shown in the formula (2).

$$
\left\{\begin{array}{l}
O_{W}(x)=\frac{3 k^{\delta}}{2\|G(x)\|(k-m)} \\
S_{W}(x)=\frac{3 G(x)\left\|F_{k}\right\|}{2 \delta}
\end{array}\right.
$$

The opportunistic weight coefficient can be obtained by the formula (3).

$$
\delta=\left\{\begin{array}{l}
\|k\|, 0 \leq j<\frac{m}{3} \\
\sqrt{k^{2}+j^{2}}, \frac{m}{3} \leq j<\frac{m}{2} \\
-\left\lceil\frac{m}{k}\right\rceil, j<0 \quad \text { or } \quad j \geq \frac{m}{2}
\end{array}\right.
$$

Here, $P_{a, b}$ represents the opportunistic wavelet video frame sequence between the multimedia mobile parameter $a$ and the multimedia sequence scrambling weight $b$. $Q_{a, b}$ represents the spatial domain transfer functions with the opportunistic wavelet, as shown in the formula (4). 


$$
\left\{\begin{array}{l}
P_{a, b}=\int_{i=1}^{k} G(i)^{\delta} d i \\
Q_{a, b}=\prod_{t \rightarrow k} F_{t} \int_{i=1}^{k} \delta G(i) d i
\end{array}\right.
$$

\section{Cooperative control of mobile multimedia crowd service}

We calculate the probability $P_{\mathrm{CS}}$ by the formula (5) to study the reason that the mobile multimedia receiver cannot correctly decode and compensate for the video frame. The jitter probability of multimedia stream is denoted by the cooperative control probability of video frames of mobile multimedia crowd service.

$$
P_{C S}=\left|\sqrt{2^{\frac{j}{k \cdot m}}-\delta}\right|
$$

Here, the distortion jitter number of the multimedia mobile video frame jitter is evaluated by expression as $\left(j /\left(k^{*} m\right)\right)$. Obviously, in $J=k=m, P_{\mathrm{CS}}$ has the linear monotone change characteristics. With the increase of distortion jitter number of video frames, the quality of mobile multimedia crowd service will be seriously reduced. This is due to the intense competition between the video frames of the multimedia distortion. Therefore, the mobile multimedia receiver can select the crowd service rejection probability and a sequence of video frames according to the competition state of the video frames.

In order to facilitate the analysis, symbol $v$ is defined as the crowd service priority of the distorted video frame, which maybe 0,1 , or 2 . Here, value 0 represents the high priority crowd service under fast moving. Value 1 represents the low speed movement of multimedia streams with high priority crowd services. Value 2 means low priority crowd services for multimedia streaming with low speed motion. The crowd service cooperative control weight $C_{\mathrm{CW}}$ of mobile multimedia video frame can be calculated by Eq. (6).

$$
C_{\mathrm{CW}}=\left\{\begin{array}{l}
m^{v}, \quad 0 \leq j<\frac{m}{3} \\
v^{m}, \quad \frac{m}{3} \leq j<\frac{m}{2} \\
\delta^{v}, \quad j<0 \quad \text { or } \quad j \geq \frac{m}{2}
\end{array}\right.
$$

Mobile multimedia crowd service cooperative control must satisfy the following requirements:

(1) The distortion of each mobile video frame in the multimedia network is compensated only once. This kind of video frame can enter the distortion video frame sequence if the transmission process is in conflict with the competition.
Table 1 Simulation parameter settings with mobility

\begin{tabular}{ll}
\hline Parameters & Value \\
\hline Time & $1000 \mathrm{~s}$ \\
Sending data rate & $125 \mathrm{Mbps}$ \\
Delay & $100 \mu \mathrm{s}$ \\
Data sending node number & 3 \\
Relay node number & 10 \\
Data receiving node number & 6 \\
Number of mobile node & 7 \\
Number of video frame & 15,890 \\
Mobile model & Random \\
\hline
\end{tabular}

(2) After the crowd service is rejected, the mobile multimedia stream is reconstructed based on the opportunistic wavelet model.

(3) Cooperative control between crowd services. Cooperative control of distortion of video frame sequences.

In the probability of failure of $P_{\mathrm{CP}}$ for video frame compensation, $P_{\mathrm{DC}}$ represents the competitive probability of a video frame, $R_{\mathrm{GS}}$ for mobile multimedia streaming based on the opportunistic to reconstruct the sequence of wavelet model, $P_{C}$ (service) represents the probability of cooperative between crowd services, and $P_{\mathrm{C}}$ (frame) represents the probability of the video frame sequence between the distortions of the cooperative control. It can be concluded that the receiver can correctly decode the mobile multimedia stream, such as the formula (7).

$$
S_{i, j, k}=\frac{m \int_{i=1}^{k} G(i)^{\delta} d i}{\int_{i=1}^{k} \delta G(i) d i \sum_{i=1}^{k}\left(1+\frac{\sqrt{P_{C}(\text { service })+P_{C}(\text { frame })}}{1-P_{\mathrm{CP}}}\right) P_{\mathrm{DC}}} R_{\mathrm{GS}}
$$

Table 2 Simulation parameter settings with static analysis

\begin{tabular}{ll}
\hline Parameters & Value \\
\hline Time & $1600 \mathrm{~s}$ \\
Jitter frequency & 10 times per second \\
Delay & $0.2 \mathrm{~ms}$ \\
Data sending node number & 3 \\
Relay node number & 10 \\
Data receiving node number & 6 \\
Number of mobile node & 7 \\
Number of video frame & 15,890 \\
Data packet number of one frame & {$[10,100]$} \\
Size of data packet & $1 \mathrm{~kb}$ \\
Sending rate of data packets & {$[1,8]$ packets per second } \\
\hline
\end{tabular}




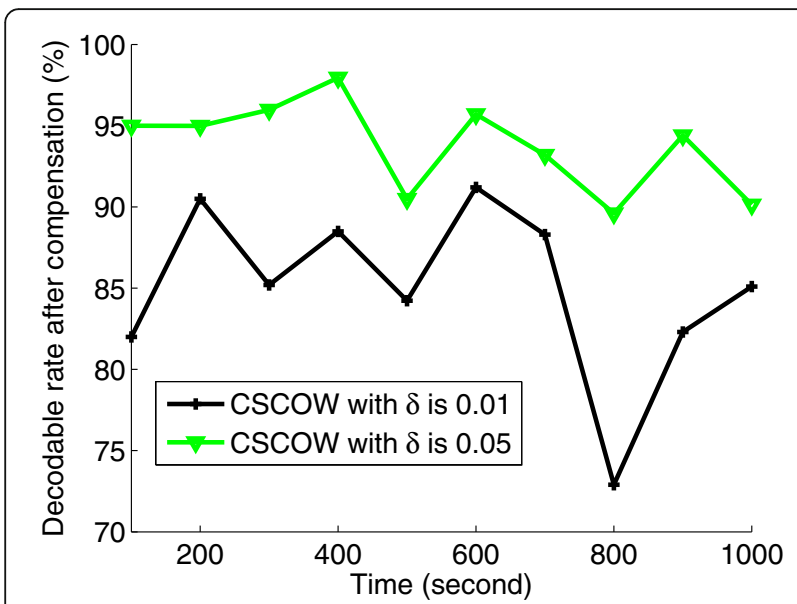

Fig. 1 Decodable rate after compensation with mobility

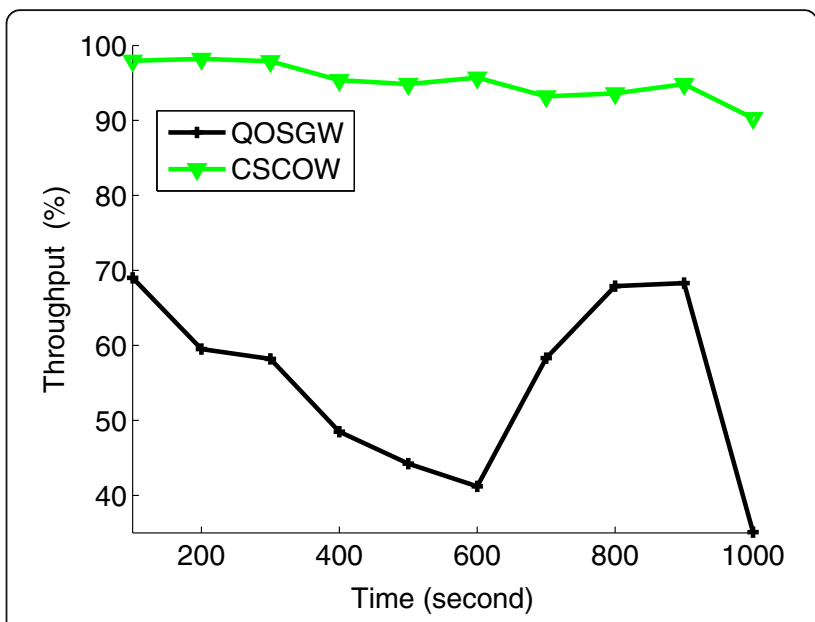

Fig. 3 Throughput with mobility

proposed mobile multimedia crowd service cooperation control protocol based on opportunistic wavelet denoted as CSCOW mechanism can provide quality assurance for multimedia mobile communication. The distortion sequence decreases with the increase of opportunistic weight. The candidate compensate ratio of video frames is reduced with the opportunistic wavelet and the crowd service cooperation control.

In the experiment, we compared the throughput and end-to-end delay of the CSCOW scheme and the multimedia service quality guarantee mechanism based on wavelet analysis denoted as QOSGW scheme, as shown in Figs. 3 and 4. Each mobile distortion video frame of CSCOW would be compensated immediately. In the transmission process, the distortion video frame sequence would be compensated for two times, which may be distorted by the competition. In addition, the crowd service of CSCOW scheme would actively reject to provide the

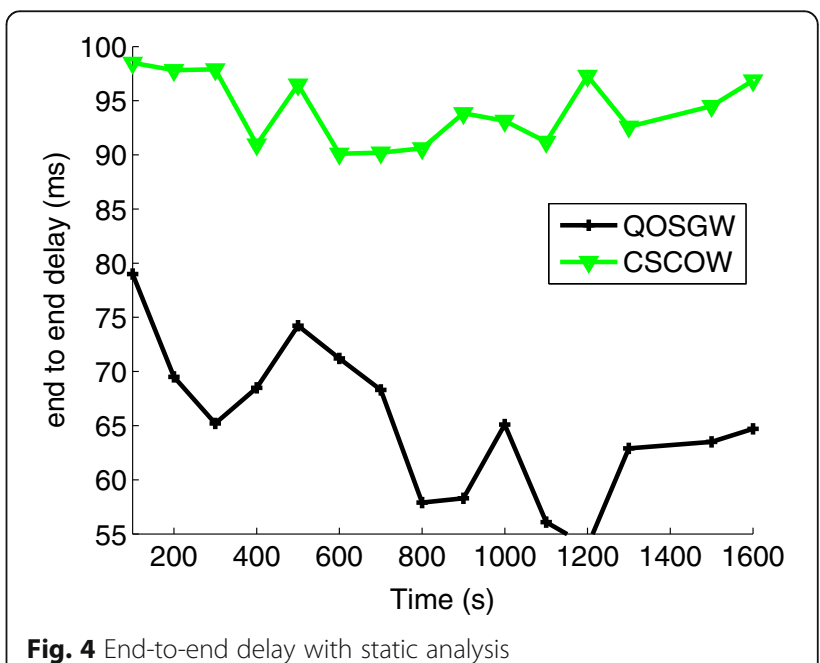

Fig. 4 End-to-end delay with static analysis

Fig. 2 Candidate compensation video frames ratio with static analysis 
services to the poor performance of the multimedia stream. After the denial of service, CSCOW would reconstruct the multimedia streaming based on the opportunistic wavelet model. In the CSCOW scheme, there is the cooperative control between crowd service and the distortion of video frame sequence. All of the above schemes can significantly improve the performance of multimedia mobile communication.

\section{Conclusions}

Based on the opportunistic wavelet model, we proposed a cooperative control protocol for multimedia mobile crowd service. The protocol can effectively improve the quality of multimedia communication and the quality of multimedia experience. On the one hand, on the basis of analyzing the delay jitter and distortion compensation of multimedia stream transmission, we give the guarantee scheme of the real-time and reliability of mobile multimedia services. At the same time, the wavelet multimedia mobile analysis model is established after the combination of the video frame resolution analysis and the wavelet scale space. On the other hand, we proposed a mobile multimedia decoding compensation crowd service scheme. In addition, a competitive and cooperative control mechanism is established between the crowd service and the distortion of the video frame sequence. Simulation results verify the effectiveness of the video frame quality assurance of mobile multimedia crowd service cooperation control protocol. At the same time, the system throughput and delay of the multimedia service quality guarantee mechanism based on the wavelet analysis are analyzed. The results show that the mobile multimedia crowd service cooperation control protocol based on opportunistic wavelet has significant advantages.

\section{Acknowledgements}

This work is supported in part by the Major research projects of Ministry of public security (201202ZDYJ017)

\footnotetext{
Authors' contributions

The study of the mobile multimedia crowd service cooperation control protocol was carried out by W-HS (Wei-Hang Shi), and the revision of wavelet model was done by W-PL (Wei-Ping Li). The simulation experiment and coding work were done by both the authors. This manuscript had been prepared and checked by both of the authors together. Both authors read and approved the final manuscript.
}

\section{Competing interests}

The authors declare that they have no competing interests.

\section{Author details}

${ }^{1}$ College of Software Technology, Zheng Zhou University, Zhengzhou, Henan 450002, China. ${ }^{2}$ School of Information Engineering, Wuhan University of Technology, Wuhan, Hubei 430070, China. ${ }^{3}$ Department of Police Technology, Railway Police College, Zhengzhou, Henan 450053, China.

Received: 22 August 2016 Accepted: 18 January 2017

Published online: 13 February 2017

\section{References}

1. IC Chang, CH Chou, HCoP-B: a hierarchical care-of prefix with BUT scheme for nested mobile networks [J]. IEEE Trans Veh Technol 58(6), 2942-2965 (2009)

2. X Xiaodong, Z Huixin, D Xun, H Yanzhao, T Xiaofeng, Z Ping, SDN based next generation mobile network with service slicing and trials [J]. China Commun 11(2), 65-77 (2014)

3. K Akkarajitsakul, E Hossain, D Niyato, Cooperative packet delivery in hybrid wireless mobile networks: a coalitional game approach [J]. IEEE Trans Mob Comput 12(5), 840-854 (2013)

4. P Rost, A Banchs, I Berberana et al., Mobile network architecture evolution toward 5G [J]. IEEE Commun Mag 54(5), 84-91 (2016)

5. AT Naman, D Taubman, JPEG2000-based scalable interactive video (JSIV) with motion compensation [J]. IEEE Trans Image Process 20(9), 2650-63 (2011)

6. MS Farid, M Lucenteforte, M Grangetto, Panorama view with spatiotemporal occlusion compensation for 3D video coding [J]. IEEE Trans Image Process 24(1), 205-19 (2015)

7. M Larson, M Soleymani, M Eskevich et al., The community and the crowd: multimedia benchmark dataset development [J]. IEEE Multimedia 19(3), 15-23 (2012)

8. CC Wu, KT Chen, YC Chang et al., Crowdsourcing multimedia QoE evaluation: a trusted framework [J]. IEEE Trans Multimedia 15(5), 1121-1137 (2013)

9. A Einarson, S Riordan, Interactive scheduling for mobile multimedia service in M2M environment [J]. Multimed Tools Appl 71(1), 235-246 (2014)

10. N Lin, Service-oriented mobile multimedia cooperative storing and delivery scheme based on opportunistic cloud coding and content-centric cloud compression [J]. EURASIP J Wirel Commun Netw 2016(1), 151 (2016)

11. K Zhang, $X$ Liang, $X$ Shen et al., Exploiting multimedia services in mobile social networks from security and privacy perspectives [J]. IEEE Commun Mag 52(3), 58-65 (2014)

12. D Kovachev, Y Cao, R Klamma, Building mobile multimedia services: a hybrid cloud computing approach [J]. Multimed Tools Appl 70(2), 977-1005 (2014)

13. D Rosário, Z Zhao, A Santos et al., A beaconless opportunistic routing based on a cross-layer approach for efficient video dissemination in mobile multimedia loT applications [J]. Comput Commun 45(1), 21-31 (2014)

14. S Saravanan, V Venkatachalam, Improving map reduce task scheduling and micro-partitioning mechanism for mobile cloud multimedia services [J]. Int J of Adv Intell Paradigms 8(2), 156-167 (2016)

15. J Liao, Q Qi, J Wang et al., A dual mode self-adaption handoff for multimedia services in mobile cloud computing environment [J]. Multimed Tools Appl 75(8), 4697-4722 (2016)

16. B Mariappan, S Ramachandran, Quality of service (QoS) enhancement for mobile multimedia applications using cross-layer (CL) mapper [J]. Int J Appl Eng Res 10(7), 18581-18592 (2015)

17. QL Yang, Q Wei, Y Huang et al., The application of multimedia messaging services via mobile phones to support outpatients: home nursing guidance for pediatric intestinal colostomy complications [J]. Chin Nurs Res 2(1), 24-27 (2015)

\section{Submit your manuscript to a SpringerOpen ${ }^{\circ}$ journal and benefit from:}

- Convenient online submission

- Rigorous peer review

- Immediate publication on acceptance

- Open access: articles freely available online

- High visibility within the field

- Retaining the copyright to your article

Submit your next manuscript at $>$ springeropen.com 